\title{
Comprehensive screening for drugs that modify radiation- induced immune responses
}

\author{
Masayuki Okumura $\mathbb{D}^{1,2}$, Junyan $\mathrm{Du}^{3,4}$, Shun-Ichiro Kageyama $\mathbb{D}^{1,5}{ }^{凶}$, Riu Yamashita ${ }^{3}$, Yumi Hakozaki ${ }^{1}$, Atsushi Motegi ${ }^{1}$, Hidehiro Hojo ${ }^{1}$, \\ Masaki Nakamura1, Yasuhiro Hirano ${ }^{1}$, Yusuke Okuma ${ }^{6}$, Hitomi S. Okuma (D) ${ }^{7}$, Katsuya Tsuchihara ${ }^{3}$ and Tetsuo Akimoto ${ }^{1,5}$
}

(c) The Author(s) 2022

BACKGROUND: Combination therapy based on radiotherapy and immune checkpoint inhibitors (ICls) was recently reported as effective for various cancers. The radiation-induced immune response (RIIR) is an essential feature in ICl-combined radiotherapy; however, the effects of drugs used concomitantly with RIIR remain unclear. We screened for drugs that can modify RIIR to understand the mutual relationship between radiotherapy and combined drugs in $\mathrm{ICl}$-combined radiotherapy.

METHODS: We established a high-throughput system with reporter gene assays for evaluating RIIR, focusing on factors acting downstream of the STING-IRF pathway, which can stimulate cancer cells, T cells, and dendritic cells. We further quantified the effects of 2595 drugs, including those approved by the Food and Drug Administration, on RIIR in vitro.

RESULTS: The reporter assay results correlated well with the expression of immune response proteins such as programmed deathligand 1. This high-throughput system enabled the identification of drugs including cytotoxic agents, molecular-targeted agents, and other agents that activate or suppress RIIR.

CONCLUSIONS: Our study provides an encyclopedic catalogue of clinically approved drugs based on their effect on RIIR. In ICls combined radiotherapy, activation of STING-IFN may improve the therapeutic effect and our result could form a biological basis for further clinical trials combining radiotherapy with ICls.

British Journal of Cancer (2022) 126:1815-1823; https://doi.org/10.1038/s41416-021-01688-0

\section{BACKGROUND}

Radiotherapy (RT) is a widely used treatment for various cancers, including lung cancer [1], and recent data revealed that RT can enhance the efficacy of immune checkpoint inhibitors (ICls) by upregulating MHC class I and programmed death-ligand 1 (PD-L1) expression in cancer cells [2]. Furthermore, a phase III trial showed that definitive chemoradiotherapy (CRT) followed by administration of durvalumab, a PD-L1 antibody, prolonged overall survival in patients with locally advanced non-small-cell lung cancer (NSCLC) $[3,4]$.

Several drugs have also been reported to modify the cancer immune response; for example, corticosteroids suppress the effects of $\mathrm{ICl}$ monotherapy $[5,6]$. It was recently reported that in combination therapy using RT, the effect of $\mathrm{ICl}$ combined with RT differed depending on the cytotoxic drug used concomitantly [7]. In CRT for NSCLC, it is possible to select and use two anticancer drugs in regimens known as platinum doublets, in which a platinum agent such as cisplatin is paired with a third-generation chemotherapy counterpart such as a taxane, pemetrexed, etoposide (ETP), vinorelbine (VNR), or 5-fluorouracil (5-FU) [8-10].
Therefore, it is important to evaluate whether cytotoxic drugs combined with RT enhance the immune response. For instance, molecular-targeted drugs such as olaparib and trametinib are likely to induce immune responses against cancer [11].

In addition to anticancer drugs, many patients with cancer are prescribed several other medicines such as COX inhibitors for antiinflammatory purposes during supportive care. Therefore, it is also essential to investigate the effects of drugs other than anticancer drugs on the immune response. However, as over 2000 drugs are currently approved, it is difficult to screen the effect of all drugs on the radiation-induced immune response (RIIR) through in vivo experiments.

Recently, radiation was shown to induce an immune reaction in the tumour tissue and surrounding immune cells via the micronucleus-forming STING-type I interferon (IFN) pathway in vitro and in vivo, which is considered as a master regulator of the cancer-immune reaction including the immune reaction in the tumour microenvironment (TME) triggered by irradiation [12]. STING activation enhances cancer antigen presentation, contributes to the priming and activation of $T$ cells, facilitates the

\footnotetext{
'Department of Radiation Oncology, National Cancer Center Hospital East, Chiba, Japan. ${ }^{2}$ Department of Radiology, Nagoya University Graduate School of Medicine, Nagoya, Aichi, Japan. ${ }^{3}$ Division of Translational Informatics, Exploratory Oncology Research and Clinical Trial Center, National Cancer Center, Chiba, Japan. ${ }^{4}$ Department of Integrated Biosciences, Graduate School of Frontier Sciences, The University of Tokyo, Chiba, Japan. ${ }^{5}$ Division of Radiation Oncology and Particle Therapy, Exploratory Oncology Research and Clinical Trial Center, National Cancer Center, Chiba, Japan. ${ }^{6}$ Department of Thoracic Oncology, National Cancer Center Hospital, Tokyo, Japan. ${ }^{7}$ Department of Breast and Medical Oncology, Clinical Research Support Office, National Cancer Center Hospital, Tokyo, Japan. ${ }^{凶}$ email: skageyam@east.ncc.go.jp
}

Received: 26 April 2021 Revised: 10 November 2021 Accepted: 23 December 2021

Published online: 19 February 2022 
trafficking and infiltration of $\mathrm{T}$ cells into tumours, and promotes the recognition and killing of cancer cells by $T$ cells [13, 14]. Notably, radiation activates the STING-IRFs-IFN pathway in cancer cells and then activates surrounding immune cells [12]. Therefore, evaluation of radiation-induced STING-IRFs-IFN pathway activation in cancer cells in vitro can provide a potential indicator of the RIIR. Hence, we established a high-throughput system based on a reporter gene assay (RGA) for easy evaluation of the RIIR and screened the effect of a library of 2595 clinically approved agents on the RIIR. We also assessed the potential underlying mechanisms of their effects on the RIIR.

\section{METHODS}

\section{Cell lines and RGA}

The A549 cell line is an NSCLC cell line that is often used in radiation experiments [15]. Its radiation resistance is moderate, and even a clinical dose of $2 \mathrm{~Gy} / \mathrm{fr}$ shows low toxicity; therefore, we used this cell line in our experiments. The A549-Dual cell line was obtained from Invitrogen (Carlsbad, CA, USA). The cell culture, luciferase assay, and secreted alkaline phosphatase (SEAP) RGA were performed according to the A549-Dual instructions (https://www.invivogen.com/a549-dual) [16].

STING-knockout A549 cells were established using The TrueCut Cas9 protein V2 and STING gRNA(CRISPR802251_SG and CRISPR802254_SG) as performed previously (unpublished).

\section{In vitro irradiation}

A549-Dual cells were cultured in 96-well plates prior to irradiation as previously described [17]. The cells were irradiated at a dose rate of $3 \mathrm{~Gy} /$ min using a Clinac iX System Linear Accelerator (Varian Medical Systems, Palo Alto, CA, USA) with an energy of 6 MV photons. The irradiation plan was performed by simulated computed tomography and pinnacle. The dose distribution was prepared in the range of D90 and irradiated at a dose rate of $600 \mathrm{cGy} / \mathrm{min}$. The cells were exposed to a total dose of $10 \mathrm{~Gy}$ in five times per week. Figure 1a shows a schematic of the cell culture and irradiation regimen.

\section{Enzyme-linked immunosorbent assay (ELISA)}

We measured the concentration of IFN- $\beta$ on day 1 (before irradiation), day 3 ( $24 \mathrm{~h}$ after $4 \mathrm{~Gy} / 2$ fractions [fr]), day 5 (24 h after $8 \mathrm{~Gy} / 4 \mathrm{fr}$ ), and day 8

b

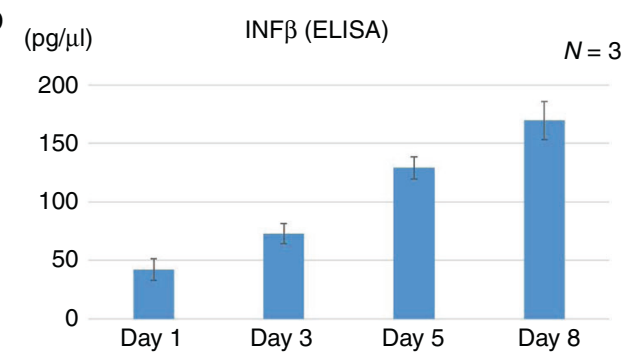

d
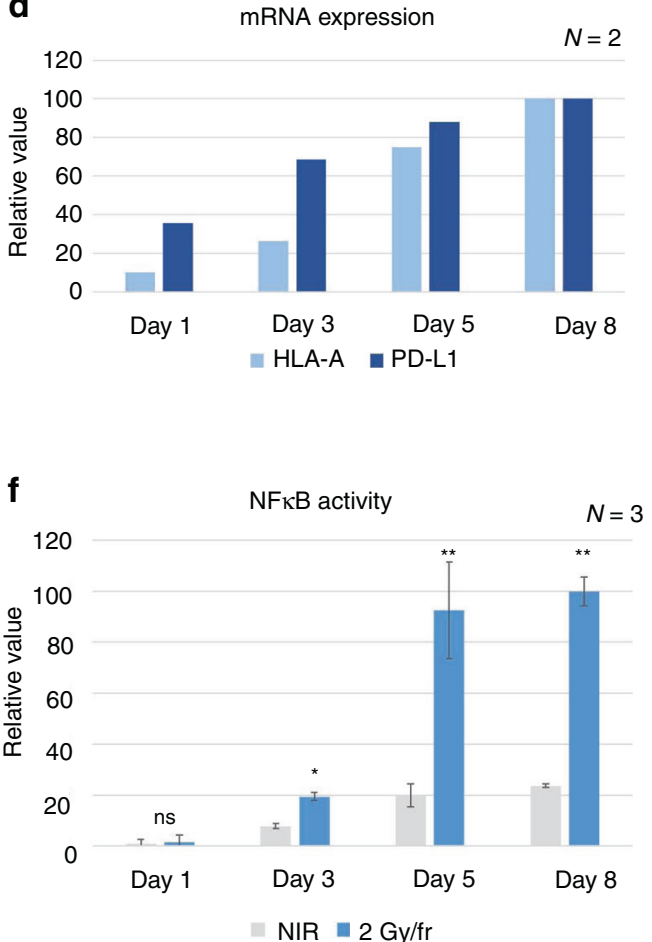

Fig. 1 Assessment of radiation-induced immune response in A549-Dual cells. a Irradiation schedule. b Quantification of IFN $\beta$ secretion during and after irradiation using ELISA. c Protein expression of STAT1Y701, STING, and PD-L1 during and after irradiation. $\mathbf{d}$ mRNA expression of HLA-A and PD-L1 during and after irradiation. Expression levels at each time point were calculated relative to that on day 8 (percentage) ( 2 days post $10 \mathrm{~Gy} / 5 \mathrm{fr}$ irradiation). e, $\mathbf{f}$ Reporter gene assay assessing ISRE and NF- $\mathrm{kB}$ activities in irradiation and non-irradiation groups. Activities at each time point were calculated relative to that on day 8 (percentage). Error bars represent STDEV. ${ }^{*} P<0.01$, ${ }^{* *} P<0.001$, ns, not significant, as determined by unpaired Student's t-test between NIR with IR group. WB western blotting, IFN interferon, ISRE interferonstimulated response element, NIR non-irradiated. 
(72 $\mathrm{h}$ after $10 \mathrm{~Gy} / 5 \mathrm{fr}$ ). The IFN- $\beta$ concentration was measured in $100 \mu \mathrm{L}$ of culture medium using Human IFN- $\beta$ ELISA Kit (R\&D Systems, Minneapolis, MN, USA) following the manufacturer's protocol.

\section{Drug screening}

Detailed information regarding the library of chemical drugs (Selleck Chemicals, Houston, TX, USA, L2000-Z383425) and screening results are listed in Supplementary Table 1. This chemical library classified drugs into categories such as cancer, inflammation, and infection and identified target molecules. Screening was performed in a 96-well plate using a semiautomatic INTEGRA VIAFLO 96 system (INTEGRA Biosciences, Tokyo, Japan). The test drug was added at a final concentration of $10 \mu \mathrm{M}$ at $1 \mathrm{~h}$ before $8 \mathrm{~Gy} / 4 \mathrm{fr}$ irradiation. Anticancer drugs, anti-inflammatory drugs, and other drugs selected in the initial screening were revalidated at concentrations of $10-0.1 \mu \mathrm{M}[18]$, and the reproducibility of the results was confirmed in at least two independent experiments. Drugs that were more toxic than the $30 \%$ inhibition concentration $\left(\mathrm{IC}_{30}\right)$ at $0.1 \mu \mathrm{M}$ were screened again at up to $1 \mathrm{nM}$. The results for the non-irradiated and irradiated groups are shown as the fold-change with respect to non-irradiated and irradiated cells treated with vehicle only, respectively. Cytotoxicity was assessed and corrected using the alamar blue assay. Concentrations at which the survival rate was $10 \%$ or less were excluded (Supplementary Table 1).

\section{Gene expression analysis}

RNA was extracted from $10^{6}$ to $10^{7}$ irradiated cancer cells using an RNeasy mini kit (QIAGEN, Hilden, Germany). The quality of the extracted RNA was assessed on an Agilent Bioanalyzer 2100 system (Agilent, Santa Clara, CA, USA), and RNA sequencing (RNA-seq) was performed (Annoroad, Beijing, China). All data were subjected to quality control filtering, trimming, and adaptor removal using the FASTQC and FASTQ toolkits (BaseSpace, Illumina, San Diego, CA, USA). Specifically, reads containing $N>10 \%$ (where $N$ indicates an undetermined base) or of low quality ( $Q$ score $\leq 5)$, which was over $50 \%$ of the total bases, were removed. All filtered sequences were aligned to the hg38 reference genome used for gene expression analyses and represented as transcripts per million calculated using RSEM software [19]. Differentially expressed genes (DEGs) were identified using the $R$ package edgeR [20] with a false discovery rate $\leq$ 0.05 . DEGs were used for Gene Ontology (GO) and Kyoto Encyclopedia of Genes and Genomes (KEGG) analyses using the iPathway Guide (ADVAITA, Ann Arbor, MI, USA) (Supplementary Tables 2 and 3) and Metascape [21] and then visualised utilising Cytoscape [22].

Principal component analysis (PCA) was performed using $R$ package plot3D, with the transcripts per million of all expressed genes in each sample used as input. A Venn diagram was constructed using the web tool InteractiVenn [23].

\section{Nuclear staining}

Cells were fixed in $4 \%$ formaldehyde for $15 \mathrm{~min}$ at $24^{\circ} \mathrm{C}$, washed with phosphate-buffered saline, and stained with $1 \mu \mathrm{g} / \mathrm{mL}$ Hoechst 33342 for $10 \mathrm{~min}$ at room temperature. Images were captured using a BZ9000 fluorescence microscope system (Keyence, Tokyo, Japan). The structures stained by Hoechst 33342 outside the nucleus were classified as micronuclei. Cells with micronuclei were counted manually in each field ( $\times 20$ magnification), and the results are expressed as the percentage of all cells within the field counted. A total of $50-70$ cells was counted in each field.

\section{Real-time polymerase chain reaction (PCR)}

The mRNA expression levels of $P D L 1$ and $M X 1$ were quantified by performing real-time PCR and using specifically designed primers. The following primers were used: PDL1 forward 5'-GGTGGTGCCGACTACAAGCGA-3', PDL1 reverse $5^{\prime}$-CCTTGGGTAGCCCTCAGCCT-3'; MX1 forward 5'-TCTGAGGAGAGCCAGAC GAT-3', MX1 reverse 5'-ACTCTGGTCCCCAATGACAG-3'; HLA-B forward $5^{\prime}$-GCGAGTCCCGAGGATGGC-3', HLA-B reverse $5^{\prime}$-TTGTAGTAGCCGCGCA GGT-3'; ACTB forward 5'-TCACCCACACTGTGCCCATCTACGA-3', ACTB reverse $5^{\prime}$-CAGCGGAACCGCTCATTGCCAATGG-3'. The mRNA expression levels were quantified based on the relative cycle threshold values for each sample and normalised to beta-actin (ACTB) expression.

\section{Western blotting (WB)}

Cells were harvested $24 \mathrm{~h}$ after exposure to the radiation doses indicated. For protein extraction, cells were scraped and washed with cold PBS, then lysed in RIPA buffer (Wako, Japan) containing a proteinase inhibitor cocktail (Sigma, P8340) for 20 min on ice and cleared by centrifugation. The protein samples were quantified by a BCA assay (Thermos), separated by SDS-PAGE (Wako, Japan), transferred to a PVDF membrane (Bio-Rad), and detected by immunoblotting with the antibodies indicated (Supplementary Table 4). More specifically, the blots were detected with ECL reagent (GE Healthcare, RPN2232) and visualised by ImageQuant LAS 4000mini (GE). Relative quantification was performed using ImageJ software, and GAPDH was used as the reference for normalisation.

\section{RESULTS}

RGA-based validation of RIIR assessment in A549-dual cells We investigated the validity of assessing the RIIR in cancer cells by performing an RGA. A549-Dual cells have been widely reported as reporter cells for IFN-stimulated response element (ISRE) and NF$\mathrm{KB}$ activity [24]. First, we irradiated the NSCLC cell line A549-Dual at a dose of $10 \mathrm{~Gy}$ in $5 \mathrm{fr}$ as a preclinical model and evaluated the RIIR (Fig. 1a). The protein expression of STATY701 and IFN- $\beta$, which have been reported as surrogates for the RIIR $[25,26]$, was assessed using ELISA and western blotting (Fig. 1b, c). Furthermore, we investigated the mRNA expression of the MHC class and PD-L1 genes that are downstream of STING; these genes directly participate in the cancer-immune response and are biomarkers of immune checkpoint inhibition [7, 27]. The expression of surrogate genes and MHC class I (HLA-A and HLA-B) and PD-L1 mRNAs was correlated with the activities of ISRE and NF-KB (Fig. 1b-f). All immune responses increased gradually at day 3 (i.e., after 4-Gy irradiation) and were maintained or increased up to day 8. GO analysis also showed that type I IFN and NF-KB signalling pathways were upregulated significantly from days 3 and 5 , respectively (Supplementary Table 2). Thus, we inferred that the results of RGA accurately reflected the RIIR in cancer cells. Finally, we confirmed in STING-knockout experiments that ISRE and NF-KB activity reflected STING activity induced by IR (Supplementary Fig. 1). Knocked out of STING significantly reduced radiation-induced ISRE and NFKB activity $\left(P=3 \mathrm{e}^{-5}\right.$ and $P=5 \mathrm{e}^{-6}$, respectively, student $t$-test).

\section{Drug screening using a library of Food and Drug Administration (FDA)-approved drugs}

To investigate the effects of chemical drugs on the RIIR, we individually treated A549-Dual cells with 2595 FDA-approved drugs $(10 \mu \mathrm{M}$ each), followed by irradiation at a dose of $8 \mathrm{~Gy} / 4 \mathrm{fr}$. As there are technical difficulties in creating an irradiated and a non-irradiated group on the same plate, the results for the nonirradiated and irradiated group are shown as the fold-change with respect to non-irradiated and irradiated cells treated with vehicle only, respectively. Cytotoxicity was assessed and corrected using the alamar blue assay. Concentrations at which the survival rate was $10 \%$ or less were excluded. Figure 2 a shows the ISRE and NF$\mathrm{KB}$ activities of the cells treated with 2595 drugs in the irradiated and non-irradiated groups. Most anticancer drugs exert effects at blood concentrations of $1-10 \mu \mathrm{M}$, whereas some function at concentrations around $0.1 \mu \mathrm{M}$ [18]. For detailed profiling, the immune response was evaluated in cancer cells treated with 233 drugs at concentrations of $0.1-10 \mu \mathrm{M}$ (Fig. 2b). Experiments were performed at least twice to verify the reproducibility of the results. Immune response modulation by given drugs differed according to the chemotherapeutic drugs used; in other words, some drugs activated, whereas others suppressed the RIIR (Fig. 2c). Specifically, the immune response was significantly increased by pemetrexed (PEM), paclitaxel (PTX) and cisplatin (CDDP) compared to irradiation alone. The immune response was maintained by docetaxel (DOC), carboplatin (CBDCA), 5-FU, and ETP. In contrast, the immune response was significantly inhibited compared to irradiation alone (by more than 50\%) in response to treatment with the pyrimidine metabolism antagonist gemcitabine (GEM), 
a

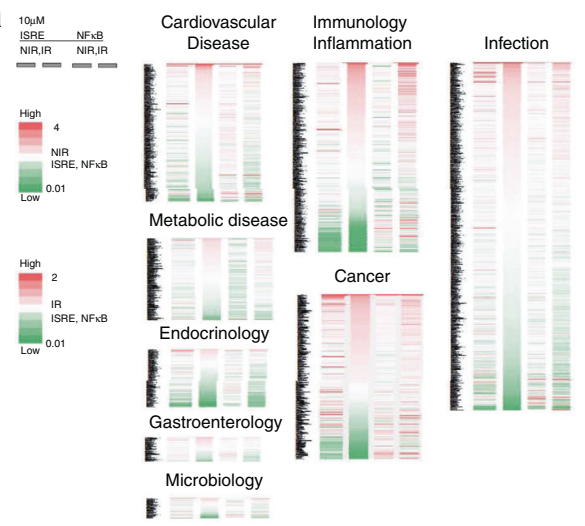
Nisease

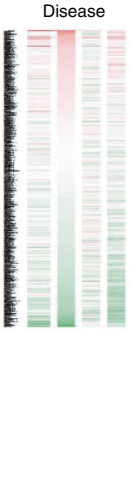

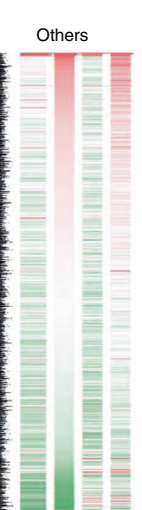

b
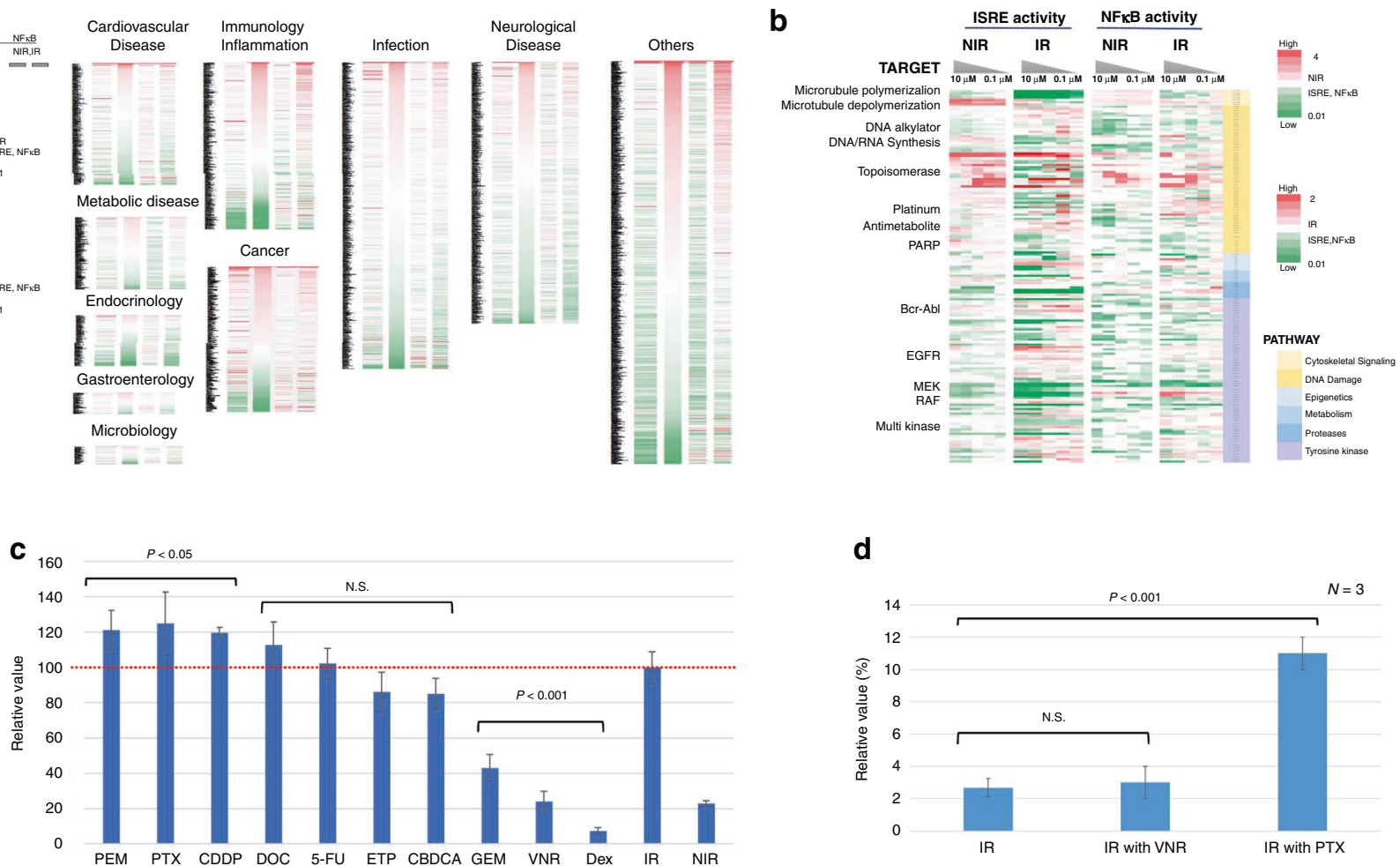

d

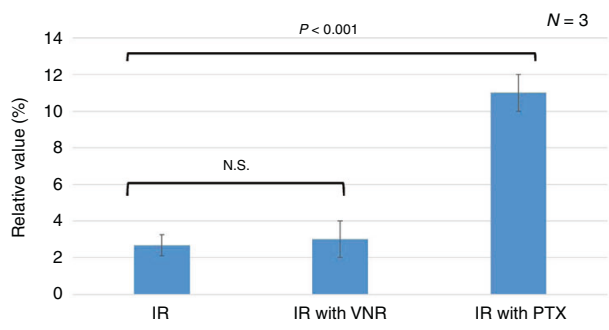

Fig. 2 Modification of the immune response by FDA-approved agents. a Heat map showing ISRE and NF- $\mathrm{KB}$ activities after administering 2595 FDA-approved agents at concentrations of $10 \mu \mathrm{M}$. Fold-change is shown with respect to the untreated group. $\mathbf{b}$ ISRE and NF- $\kappa B$ activities after administering 233 agents associated with cancer at a concentration range of $10-0.1 \mu \mathrm{M}$. Fold-change is shown with respect to the untreated group. c ISRE activities after administering the drugs to non-small cell lung cancer cells irradiated at $8 \mathrm{~Gy} / 4 \mathrm{fr}$. Values are expressed relative to the activity of the IR-alone (drug-free) group as 100 . d Percentage of micronuclei formation after administering vinorelbine or paclitaxel with or without $8 \mathrm{~Gy} / 4 \mathrm{fr}$ irradiation. Error bars represent STDEV. ns, not significant, as determined by unpaired Student's $t$-test between IR-alone with IR with the drug group. ISRE interferon-stimulated response element, NIR non-irradiated, IR irradiated, PEM pemetrexed, PTX paclitaxel, CDDP cisplatin, DOC docetaxel, CBDCA carboplatin, ETP etoposide, GEM gemcitabine, VNR vinorelbine, Dex dexamethasone.

microtubule polymerisation inhibitor such as VNR, and a corticosteroid such as dexamethasone (Dex) (Fig. 2c). The validity of the RGA results was evaluated by comparing the changes in mRNA expression levels of $M X 1$, which is regulated by the ISRE promoter, and the expression of PDL1 and MHC class I (HLA-A and HLA-B), which are regulated by STING. The RGA results correlated well with the mRNA expression levels of $M X 1$ and $P D L 1$ observed in three independent real-time polymerase chain reaction experiments (Supplementary Fig 2A). The RGA results also correlated with the levels of MHC class I mRNA expression in two independent RNA-seq (Supplementary Fig 2B).

\section{Transcriptome analysis by RNA-seq}

Next, we investigated the mechanisms underlying the differential modifications of the RIIR according to the drugs used. As described above, the microtubule depolymerisation inhibitors PTX and DOC resulted in stronger activation of the RIIR than that observed with the microtubule polymerisation inhibitor VNR when combined with irradiation. Micronuclei formation, which reflects activation of the STING-type I IFN pathway, was more prominent following PTX treatment than after VNR treatment when combined with radiation (Fig. $2 \mathrm{~d}$ and Supplementary Fig $3 \mathrm{~A}$ ). Furthermore, we found that CDK1 and Aurora A inhibitors, which arrest the cell cycle before metaphase, attenuated the RIIR. In contrast, Aurora B inhibitors, which arrest the cycle from metaphase to telophase, did not significantly influence the RIIR (Supplementary Fig 3B).

Next, we analysed the effect of chemotherapeutic drugs that are typically used with RT as platinum doublets and Dex in CRT for
NSCLC on the gene expression profile by performing RNA-seq. The gene expression profiles were analysed and compared in the following groups: non-irradiation, irradiation alone, and irradiation combined with each drug. For each drug, $3 \mu \mathrm{M}$ (PEM, ETP, 5-FU, OLA), $2 \mu \mathrm{M}$ (PTX, DOC), $1 \mu \mathrm{M}$ (GEM, CDDP, CBDCA), and $0.1 \mu \mathrm{M}$ (VNR, DEX) were adopted because these concentrations were the $\mathrm{IC}_{50}$ dose in A549 cells and within the range of the blood concentration in patients. The total gene expression profile is shown PCA plot (Fig. 3a), and as the KEGG pathway (Fig. 3b) and GO (Fig. 3c and Tables 1 and 2, and Supplementary Table 3) analysis results. In KEGG enrichment analysis for signal pathways, the p53 signalling pathway (hsa04115) was significantly enriched in combination with most drugs (Fig. 3b). In cluster analysis on the KEGG pathway, irradiation alone and the combination with PEM, CDDP, and CBDCA formed the same cluster. PTX and DOC also formed other clusters. Table 2 and Supplementary Table 3 showed the outline of the biological process induced by irradiation in combination with each drug and 14 common biological processes (Fig. 3c), including type I IFN signalling except for GEM, VNR, and Dex (Table 1 and Supplementary Table 3), were confirmed. We also show the expression profile of genes involved in type I IFN signalling in a heat map (Fig. 3d).

The molecular-targeted drugs analysed enhanced or suppressed the RIIR, similar to the effects of chemotherapeutic drugs. For example, osimertinib which specifically inhibits epidermal growth factor receptor (EGFR), entrectinib which inhibits protooncogene tyrosine-protein kinase/tropomyosin receptor kinase (ROS1/NTRK), crizotinib which inhibits MET/anaplastic lymphoma kinase (MET/ALK), and olaparib which inhibits poly ADP ribose 
a

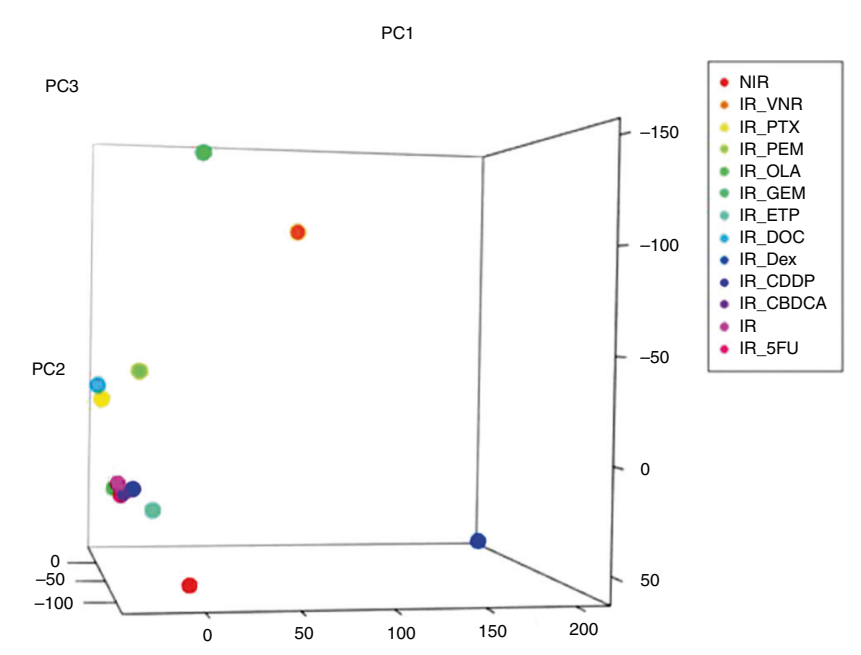

C

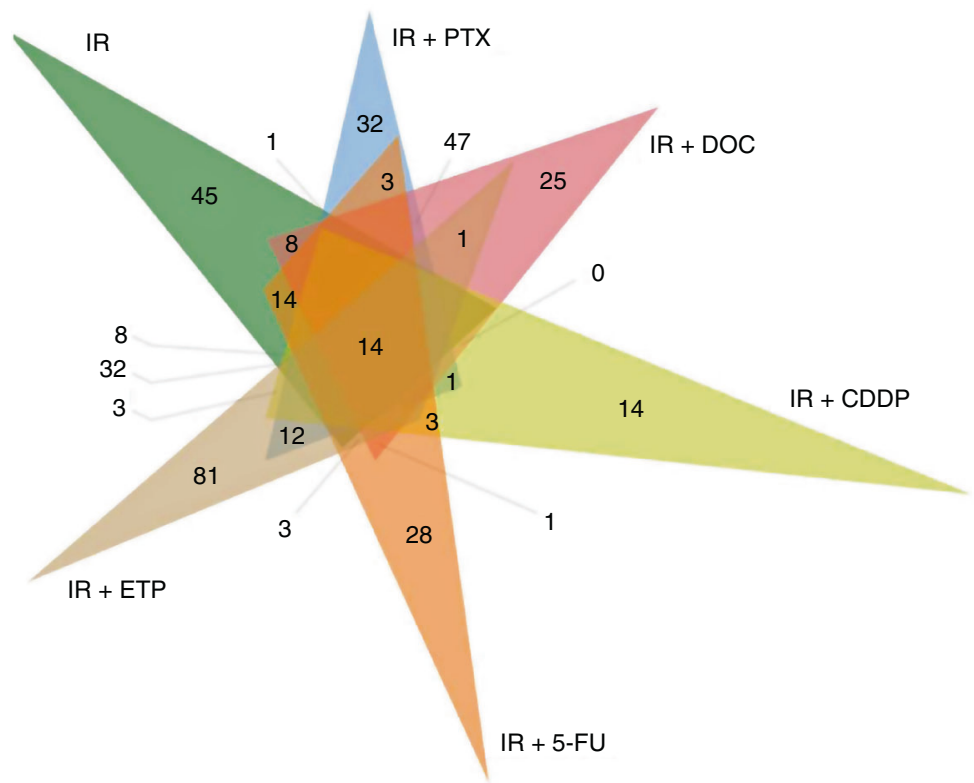

b

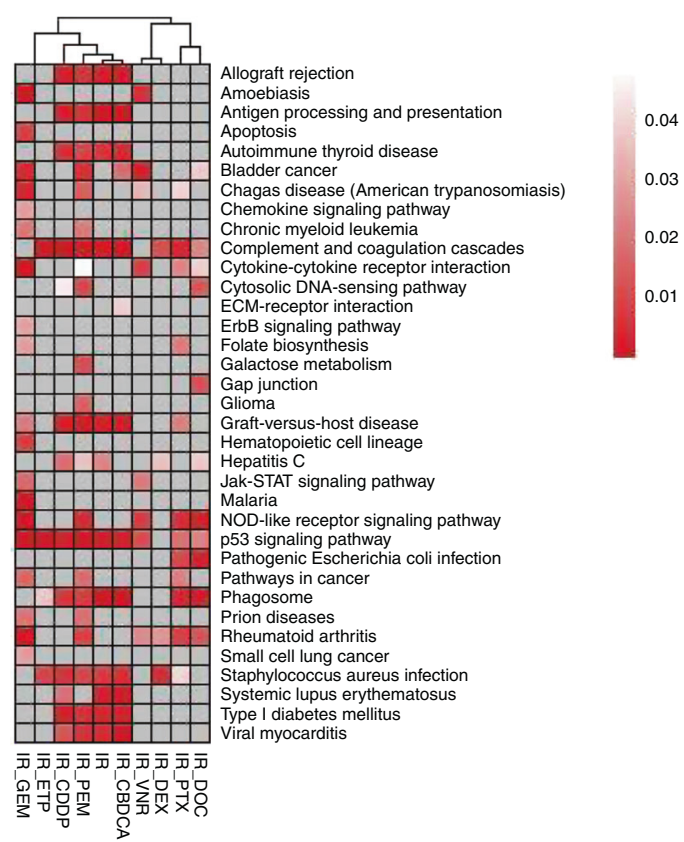

d

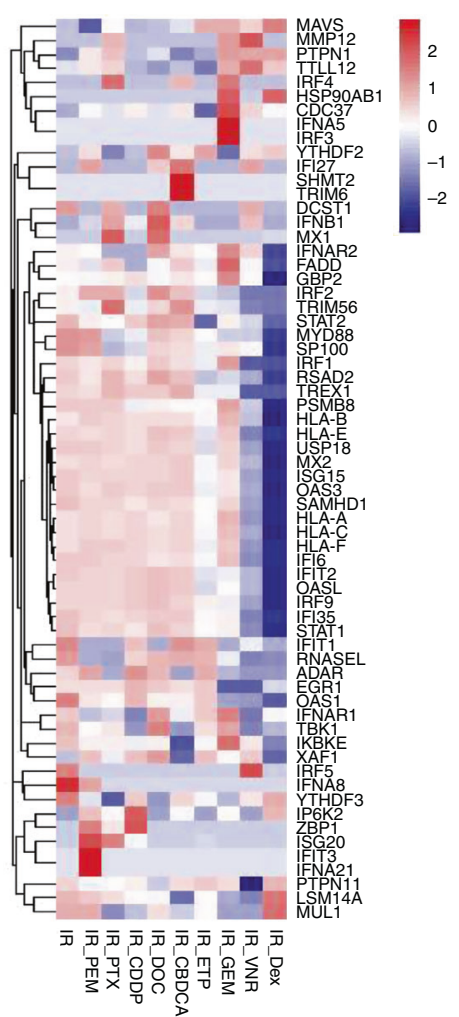

Fig. 3 Transcriptome analysis of the radiation-induced gene expression profile. a PCA plot analysis in NIR, IR, IR with PEM, PTX, CDDP, DOC, CBDCA, ETP, GEM, VNR, 5-FU, OLA, and Dex groups. b KEGG enrichment analysis for signal pathway in IR, IR with PEM, PTX, CDDP, DOC, CBDCA, ETP, GEM, VNR, and Dex groups. c Venn diagram of biological process in IR, IR with DOC, PTX, CDDP, 5-FU, and ETP groups. d Heat map of gene expression profiles in IR, IR with PEM, PTX, CDDP, DOC, CBDCA, ETP, GEM, VNR, and Dex groups. NIR non-irradiated, IR irradiated, IR with PEM pemetrexed, PTX paclitaxel, CDDP cisplatin, DOC docetaxel, CBDCA, carboplatin, ETP etoposide, GEM gemcitabine, VNR vinorelbine, 5-FU 5-fluorouracil, OLA olaparib, Dex dexamethasone.

polymerase (PARP) enhanced or maintained the RIIR, whereas dabrafenib which inhibits proto-oncogene B-Raf (BRAF), trametinib which inhibits mitogen-activated protein kinase (MEK), and vorinostat which inhibits histone deacetylase (HDAC) significantly reduced the RIIR (Supplementary Fig. 4). Anti-inflammatory and immunological drugs such as corticosteroids, JAK inhibitors, antimetabolites, and microtubule polymerisation inhibitors suppressed the RIIR (Supplementary Fig. 5). In contrast, COX inhibitors, which are often used in patients with cancer, had little effect on the RIIR (Supplementary Fig. 5). 
Table 1. Enrichment analysis of genes upregulated in response to drug-combined radiation in the non-irradiated group.

\begin{tabular}{llll}
$\begin{array}{l}\text { Drug } \\
\text { IR }\end{array}$ & $\begin{array}{l}\boldsymbol{P} \text {-value } \\
1.85 \mathrm{E}-26\end{array}$ & $\begin{array}{l}\text { Q-value } \\
2.06 \mathrm{E}-23\end{array}$ & $\begin{array}{l}\text { Gene ID (type I interferon signalling pathway, GO: } \\
\text { IFI6/ISG15/HLA-B/HLA-A/HLA-C/IFIT1/IFIT3/OASL/IFITM1/IFI27/IFIT2/OAS3/MX1/STAT1/IFI35/HLA-F/HLA-E/ } \\
\text { OAS1/ADAR/XAF1/STAT2/PSMB8 }\end{array}$ \\
\hline PEM & $4.78 E-14$ & $4.82 E-11$ & HLA-B/ADAR/IFI27/IFIT3/OASL/IFIT2/HLA-C/ISG15/IFIT1/HLA-F/IFI6/HLA-A/STAT1 \\
\hline PTX & $5.59 E-06$ & 0.000695 & HLA-B/IFIT3/IFIT2/OASL/HLA-F/ISG15 \\
\hline CDDP & $2.46 E-22$ & $2.24 E-19$ & ADAR/HLA-B/IFIT3/OASL/ISG15/IP6K2/IFIT2/HLA-C/IFI6/IFI27/XAF1/STAT1/IFIT1/HLA-A/MX1/HLA-F/OAS3 \\
\hline DOC & $1.13 E-11$ & $6.69 E-09$ & IFIT3/HLA-B/OASL/IFIT2/ADAR/IP6K2/IFIT1/ISG15/IFI27/STAT1 \\
\hline CBCDA & $1.31 E-23$ & $1.46 E-20$ & $\begin{array}{l}\text { ISG15/IFI6/HLA-B/HLA-C/HLA-A/IFIT3/OASL/IFI27/IFIT1/IFIT2/IFITM1/MX1/OAS3/STAT1/HLA-F/HLA-E/OAS1/ } \\
\text { IFI35/XAF1/TREX1 }\end{array}$ \\
\hline ETP & 0.000337 & 0.005645 & IFI6/HLA-A/ADAR/HLA-B/HLA-C \\
\hline GEM & n.s. & & \\
\hline VNR & n.s. & & \\
\hline Dex & n.s. & & \\
\hline
\end{tabular}

The dynamics of type I interferon signal analysis are shown.

IR irradiated, PEM pemetrexed, PTX paclitaxel, CDDP cisplatin, DOC docetaxel, CBDCA carboplatin, ETP etoposide, GEM gemcitabine, VNR vinorelbine, Dex dexamethasone, n.s. not significant.

\section{DISCUSSION}

We used a high-throughput RGA system to quantify the effects of clinically used drugs on the RIIR. Many drugs that activate, maintain, or suppress the RIIR were screened (Supplementary Table 1). Below, we discuss the biological basis and clinical importance of the findings of the current study.

The RIIR can be either STING-dependent or independent, but most responses require gene expression via the IRF-dependent ISRE promoter or NF-KB promoter $[12,26]$. Our high-throughput RGA system was validated by the results showing that ISRE and NF$\mathrm{KB}$ activity correlated with IFN $\beta$ secretion and mRNA expression levels of immune-related factors, including $M X 1$ regulated by the ISRE promoter and PDL1, HLA-A, and HLA-B regulated by STING (Fig. 1 and Supplementary Fig. 2 and Supplementary Table 2). Furthermore, using the STING-knockout cell line, we confirmed that approximately $95 \%$ of radiation-induced ISRE activity and $70 \%$ of NF-KB activity was derived from STING (Supplementary Fig. 1).

The drug screening results revealed that chemotherapeutic drugs used in combination with RT maintained or suppressed the RIIR, depending on their targets. Microtubule-targeting drugs, including PTX, DOC, and VNR, had particularly distinct effects, despite being M-phase inhibitors (Fig. 2b, c). These drugs can be selected as partners of platinum doublets and are used in combination with ICls. The large difference in activation or suppression of the STING-IRFs-IFN pathway according to each drug is an interesting finding, as the efficacy of the combination of ICls and RT may be influenced via modification of the RIIR.

Chemotherapeutic drugs induce micronuclei formation, although the degree of this induction differs according to the targets $[28,29]$. In addition, antimitotic drugs can cause micronuclei formation, induce a cancer immune reaction, and activate lymphocytes by activating the STING-IRFs-IFN in cancer cells both in vitro and in vivo [30]. Our study identified the RIIRmodulating effects of these antimitotic drugs. For example, M-phase inhibitors (agents that target processes before the metaphase), such as CDK1 inhibitor, Aurora A, and VNR, strongly suppressed the RIIR. In contrast, drugs that target processes after metaphase, such as Aurora B, DOC, and PTX, maintained or enhanced the RIIR (Supplementary Fig. 3B). The formation of micronuclei differed significantly in response to VNR and PTX treatment, which may differentially modulate the RIIR.

The large difference in the RIIR, mediated by different cytotoxic drugs commonly used in CRT for NSCLC, was the most interesting finding of the current study. Platinum-based chemotherapy, including platinum doublet, has been established as a standard chemotherapy regimen of CRT for NSCLC [8-10]; however, its routine use remains controversial in combination with ICls [31] because of the effect on the TME. Post-hoc analysis of the PACIFIC trial suggested that the clinical outcomes were slightly better in patients who received CRT with cisplatin-based chemotherapy than in those who received CRT with carboplatin-based chemotherapy [7]. Our results based on the high-throughput RGA system also revealed that the effect of cisplatin on the RIIR was higher than that of carboplatin, suggesting that chemotherapeutic drugs differentially affect the RIIR.

In gene expression analysis, PCA plots, KEGG pathway analysis, and GO analysis identified gene expression profiles and biological processes in irradiated cells treated with each drug (Fig. 3a, c). The immune reaction was commonly regulated via the IFN-dependent pathway (Fig. 3b and Table 1 and Supplementary Table 3). This finding indicates that the type I IFN pathway is a common and effective therapeutic target for platinum doublet combination RT.

Additionally, drugs specific for molecular targets, such as inhibitors of EGFR, PARP, BRAF, MEK, ALK/ROS, and HDAC, are part of a promising therapeutic approach for metastatic NSCLC and exhibited distinct effects on the RIIR (Supplementary Fig. 4). Furthermore, we identified several anti-inflammatory drugs that suppressed the anticancer immune response induced by irradiation (Supplementary Fig. 5). Although corticosteroids mainly affect the activity and function of lymphocytes and neutrophils and reduce the effects of $\mathrm{ICI}[5,6]$, we observed that corticosteroids also modified the RIIR. Moreover, JAK inhibitors, MTX, and colchicine, which are used to treat autoinflammatory diseases, reduced the RIIR, although COX inhibitors had little effect. Importantly, COX inhibition and the IFN response have been reported to correlate with radiosensitivity [32]. However, recent studies reported that the radiation-induced IFN response is important for the abscopal effect, and the effect of radiationinduced IFN on cancer cells remains controversial regarding whether this effect is favourable for cancer treatment [33].

As the ISRE activity per surviving cell was measured using the alamar blue assay in our experiment, radiosensitivity requires further analysis. We discussed only the immune response per cell and the potential for radiation therapy with $\mathrm{ICls}$.

Herein, we focused only on the immune response of cancer cells to irradiation, which is a limitation of this study. It is well-known that immune cells, including lymphocytes, are influenced or inactivated by RT or CRT [34], indicating that the effect of RT on immune cells influences the overall immune response of cancer. However, it has been reported that radiation first activates the 


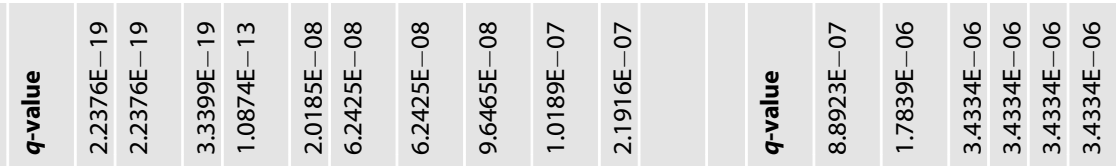

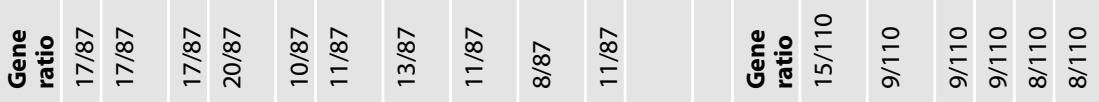

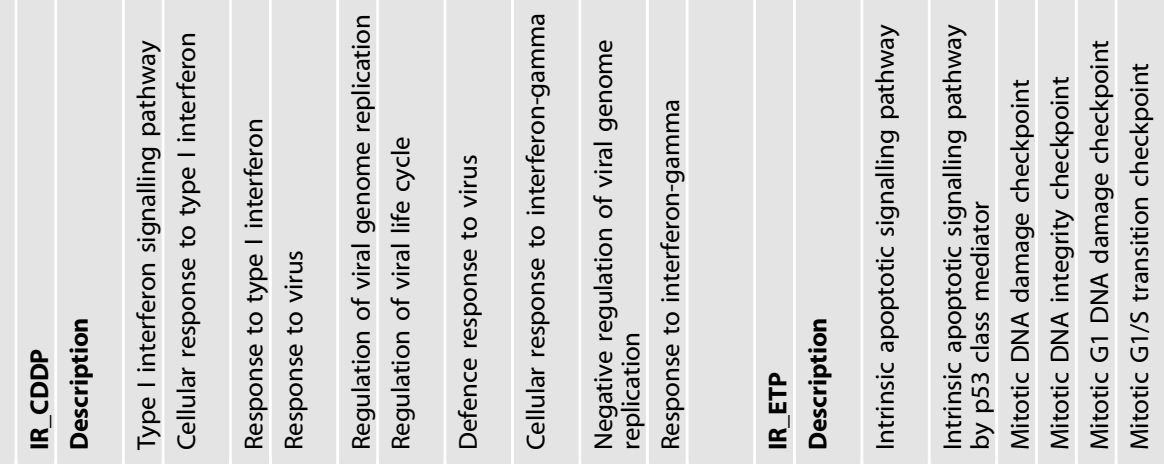

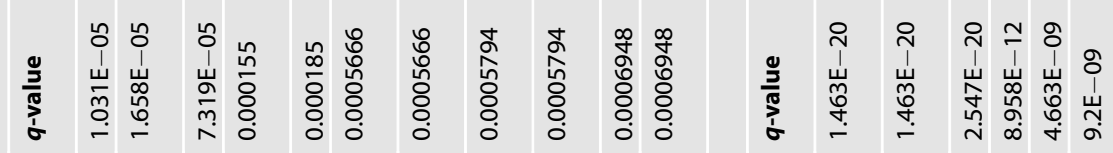

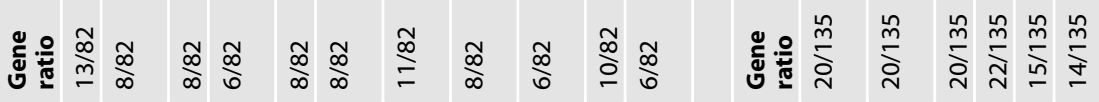

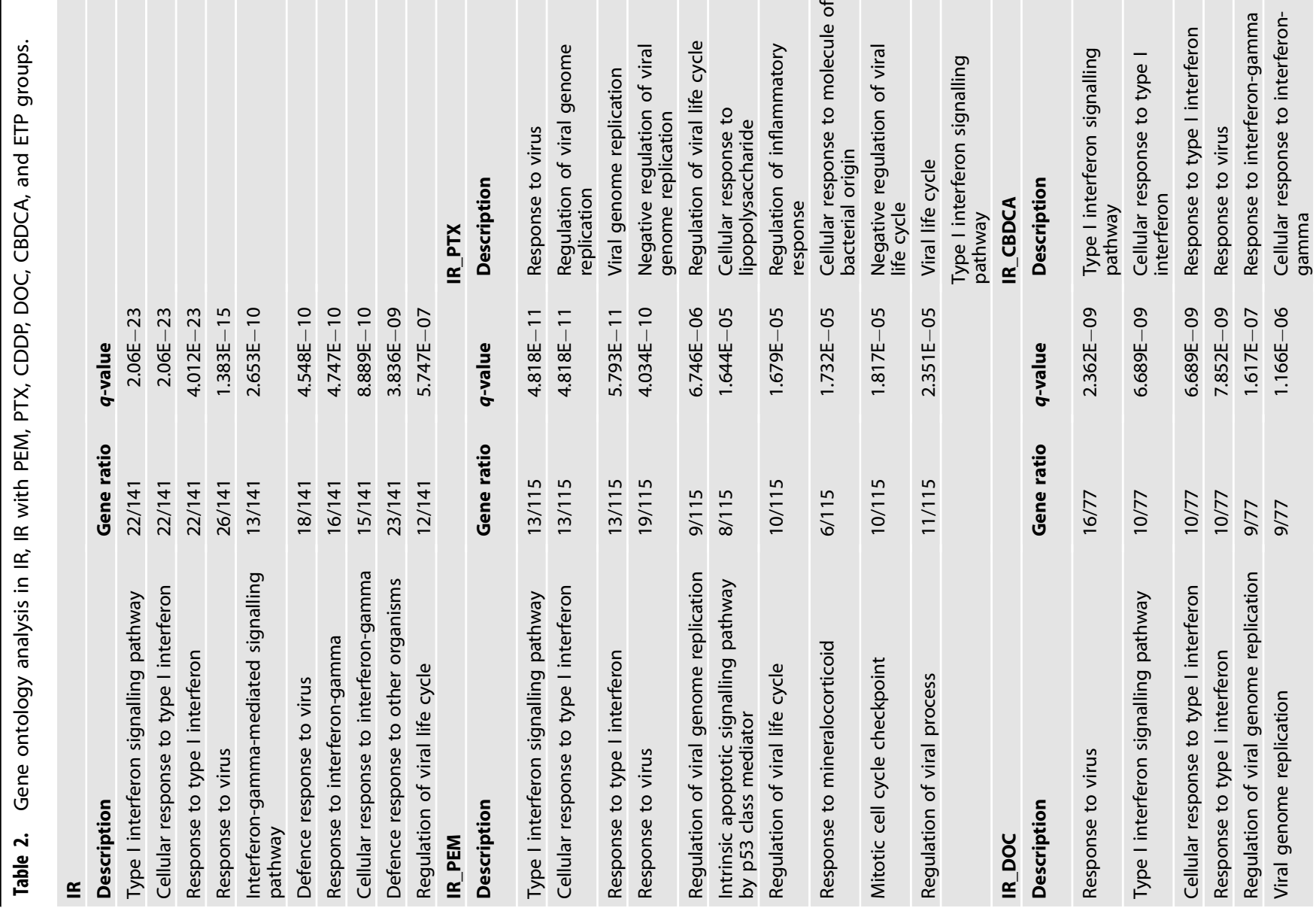


STING-IRFs-IFN pathway in cancer cells, and then in surrounding immune cells [12]. Therefore, evaluation of radiation-induced STING activity in cancer cells in vitro may reflect the RIIR in cancer cells. It has been reported that olaparib, a PARP inhibitor, activates tumour cells and surrounding lymphocytes [35], whereas steroids reduce the cancer immune response $[5,6]$. Our system successfully reproduced the immune response enhancement mediated by olaparib and immune response suppression mediated by steroids, suggesting that this high-throughput RGA system can be used to evaluate the immune status while considering the TME. Furthermore, our screening system required stably established RGA cell lines, and A549 is the only NSCLC cell line that has been established. Therefore, we only used A549 cells in this study for initial screening; however, further studies are needed to investigate various NSCLC cell lines such as EGFR and EML-ALK mutant cell lines.

Although TME analysis of cancer tissue after irradiation or clinical data analysis of $\mathrm{ICl}$-combined RT can provide more important information, pathological analysis and animal experiments are costly and time-consuming, and it is difficult to investigate the effects of more than 2000 approved drugs. Our high-throughput system is advantageous for providing primary results related to the effects of approved drugs on the RIIR in cancer cells. Our highthroughput system is complementary to TME analysis and can facilitate the advancement of ICl-combined RT. Further studies are needed to determine whether the differences in STING activation or suppression of cytotoxic and other drugs observed in this study affect the outcome of ICl combined with RT. CRT followed by PD-L1 antibody treatment for NSCLC began only two years ago, and data are currently insufficient for retrospective analysis but may be possible in the near future. Our findings would be useful for future retrospective analysis.

In summary, we established a high-throughput system for easily detecting the RIIR with STING activity and comprehensively investigated the effects of 2595 FDA-approved, clinically used drugs on RIIR in vitro using a high-throughput RGA system that can quantify the STING activity, which is an initial trigger of the RIIR. These results provide an encyclopedic catalogue of clinically approved drugs based on the effect on the RIIR. This catalogue can form the biological basis for further clinical trials of combining RT with ICls.

\section{DATA AVAILABILITY}

The datasets relevant to the current study are available from the corresponding author on reasonable request.

\section{REFERENCES}

1. Baskar R, Lee KA, Yeo R, Yeoh KW. Cancer and radiation therapy: Current advances and future directions. Int J Med Sci. 2012;9:193-9.

2. Daly ME, Monjazeb AM, Kelly K. Clinical trials integrating immunotherapy and radiation for non-small-cell lung cancer. J Thorac Oncol. 2015;10:1685-93.

3. Antonia SJ, Villegas A, Daniel D, Vicente D, Murakami S, Hui R, et al. Durvalumab after chemoradiotherapy in stage III non-small-cell lung cancer. N. Engl J Med. 2017;377:1919-29.

4. Antonia SJ, Villegas A, Daniel D, Vicente D, Murakami S, Hui R, et al. Overall survival with durvalumab after chemoradiotherapy in Stage III NSCLC. N. Engl J Med. 2018;379:2342-50.

5. Arbour KC, Mezquita L, Long N, Rizvi H, Auclin E, Ni A, et al. Impact of baseline steroids on efficacy of programmed cell death-1 and programmed death-ligand 1 blockade in patients with non-small-cell lung cancer. J Clin Oncol. 2018;36:2872-8.

6. Coutinho $A E$, Chapman KE. The anti-inflammatory and immunosuppressive effects of glucocorticoids, recent developments and mechanistic insights. Mol Cell Endocrinol. 2011;335:2-13.

7. Gray JE, Villegas A, Daniel D, Vicente D, Murakami S, Hui R, et al. three-year overall survival with durvalumab after chemoradiotherapy in Stage III NSCLC—update from PACIFIC. J Thorac Oncol. 2020;15:288-93.

8. Glatzer M, Elicin O, Ramella S, Nestle U, Putora PM. Radio(chemo)therapy in locally advanced nonsmall cell lung cancer. Eur Respiratory Rev. 2016;25:65-70. 
9. National Comprehensive Cancer Network. Non-small cell lung cancer (version 6.2020). https://www.nccn.org/professionals/physician_gls/pdf/nscl.pdf. Accessed 31 August, 2020.

10. Eberhardt WEE, De Ruysscher D, Weder W, Le Péchoux C, De Leyn P, Hoffmann H, et al. 2nd ESMO Consensus Conference in Lung Cancer: Locally advanced stage III non-small-cell lung cancer. Ann Oncol. 2015;26:1573-88.

11. Vikas P, Borcherding N, Chennamadhavuni A, Garje R. Therapeutic potential of combining PARP inhibitor and immunotherapy in solid tumors. Front Oncol. 2020;10:570.

12. Grassberger C, Ellsworth SG, Wilks MQ, Keane FK, Loeffler JS. Assessing the interactions between radiotherapy and antitumour immunity. Nat Rev Clin Oncol. 2019;16:729-45.

13. Barber GN. STING: Infection, inflammation and cancer. Nat Rev Immunol. 2015;15:760-70

14. Zhu $Y$, An $X$, Zhang $X$, Qiao $Y$, Zheng T, Li X. STING: a master regulator in the cancer-immunity cycle. Mol Cancer. 2019;18:1-15.

15. Lee DE, Alhallak K, Jenkins SV, Vargas I, Greene NP, Quinn KP, et al. A radiosensitizing inhibitor of HIF-1 alters the optical redox state of human lung cancer cells in vitro. Sci Rep. 2018;81:8815.

16. Bos $S$, Viranaicken W, Frumence $E$, Li G, Desprès $P$, Zhao RY, et al. The envelope residues $E 152 / 156 / 158$ of zika virus influence the early stages of virus infection in human cells. Cells. 2019;8:E1444.

17. Hojo H, Dohmae T, Hotta K, Kohno R, Motegi A, Yagishita A, et al. Difference in the relative biological effectiveness and DNA damage repair processes in response to proton beam therapy according to the positions of the spread out Bragg peak. Radiat Oncol. 2017;12:111.

18. Liston DR, Davis M. Clinically relevant concentrations of anticancer drugs: a guide for nonclinical studies. Clin Cancer Res. 2017;23:3489-98.

19. Li B, Dewey CN. RSEM: accurate transcript quantification from RNA-Seq data with or without a reference genome. BMC Bioinformatics. 2011;12:323.

20. McCarthy DJ, Chen Y, Smyth GK. Differential expression analysis of multifactor RNA-Seq experiments with respect to biological variation. Nucleic Acids Res. 2012;40:4288-97.

21. Zhou Y, Zhou B, Pache L, Chang M, Khodabakhshi AH, Tanaseichuk O, et al. Metascape provides a biologist-oriented resource for the analysis of systemslevel datasets. Nat Commun. 2019;10:1523.

22. Shannon P, Markiel A, Ozier O, Baliga NS, Wang JT, Ramage D, et al. Cytoscape: a software environment for integrated models of biomolecular interaction networks. Genome Res. 2003;13:2498-504.

23. Heberle H, Meirelles VG, da Silva FR, Telles GP, Minghim R. InteractiVenn: a webbased tool for the analysis of sets through Venn diagrams. BMC Bioinformatics. 2015;16:169.

24. Tissari J, Sirén J, Meri S, Julkunen I, Matikainen S. IFN-a enhances TLR3-mediated antiviral cytokine expression in human endothelial and epithelial cells by upregulating TLR3 expression. J Immunol. 2005;174:4289-94.

25. Harding SM, Benci JL, Irianto J, Discher DE, Minn AJ, Greenberg RA. Mitotic progression following DNA damage enables pattern recognition within micronuclei. Nature. 2017;548:466-70.

26. Shevtsov M, Sato $H$, Multhoff $G$, Shibata A. Novel approaches to improve the efficacy of immuno-radiotherapy. Front Oncol. 2019;9:156.

27. Rodig SJ, Gusenleitner D, Jackson DG, Gjini E, Giobbie-Hurder A, Jin C, et al. MHC proteins confer differential sensitivity to CTLA-4 and PD-1 blockade in untreated metastatic melanoma. Sci Transl Med. 2018;10:3342.

28. Stopper H, Müller SO. Micronuclei as a biological endpoint for genotoxicity: a minireview. Toxicol Vitr. 1997;11:661-7.

29. Mitchison TJ, Pineda J, Shi J \& Florian S. Is inflammatory micronucleation the key to a successful anti-mitotic cancer drug? Open Biol. 2017;7:170182.

30. Lohard S, Bourgeois N, Maillet L, Gautier F, Fétiveau A, Lasla H, et al. STING dependent paracriny shapes apoptotic priming of breast tumors in response to anti-mitotic treatment. Nat Commun. 2020;11:259.

31. Amrein MA, Bührer ED, Amrein ML, Li Q, Rothschild S, Riether C, et al. Chemotherapy negatively impacts the tumor immune microenvironment in NSCLC: an analysis of pre- and post-treatment biopsies in the multi-center SAKK19/09 study. Cancer Immunol Immunother. https://doi.org/10.1007/s00262-020-02688-4. 2020.

32. Cheki M, Yahyapour R, Farhood B, Rezaeyan A, Shabeeb D, Amini P, et al. COX-2 in radiotherapy: a potential target for radioprotection and radiosensitization. Curr Mol Pharmacol. 2018;11:173-83.

33. Yamazaki T, Kirchmair A, Sato A, Buqué A, Rybstein M, Petroni G, et al. Mitochondrial DNA drives abscopal responses to radiation that are inhibited by autophagy. Nat Immunol. 2020;21:1160-71.

34. Pike LRG, Bang A, Mahal BA, Taylor A, Krishnan M, Spektor A, et al. The impact of radiation therapy on lymphocyte count and survival in metastatic cancer patients receiving PD-1 immune checkpoint inhibitors. Int J Radiat Oncol Biol Phys. 2019;103:142-51.

35. Ding L, Kim HJ, Wang Q, Kearns M, Jiang T, Ohlson CE, et al. PARP inhibition elicits STING-dependent antitumor immunity in Brca1-deficient ovarian cancer. Cell Rep. 2018;25:2972-2980.e5.

\section{ACKNOWLEDGEMENTS}

We appreciate the help provided by radiological technologists and medical physicists at the National Cancer Center Hospital East.

\section{FUNDING}

This study was funded in part by Grants-in-Aid for Scientific Research KAKENHI [grant number 18K07740], Japan Agency for Medical Research and Development (AMED) [grant numbers 18ck0106210h0003, 19ck0106485h001], and National Cancer Center Research and Development Fund [31-A-10]. The funders had no role in the study design and collection, analysis, and interpretation of data, writing of the manuscript, and decision to submit the article for publication.

\section{AUTHOR CONTRIBUTIONS}

$\mathrm{S}-\mathrm{IK}, \mathrm{OM}, \mathrm{JD}, \mathrm{MN}, \mathrm{HH}, \mathrm{HY}$, and $\mathrm{AM}$ conducted the experiments and radiotherapy. OM, S-IK, YO, TA, AO, and KT wrote the manuscript. JD and YR performed the bioinformatics analysis. OM, JD, and S-IK performed the data analysis and prepared the figures for the manuscript.

\section{COMPETING INTERESTS}

The authors declare no competing interests.

\section{ETHICS APPROVAL AND CONSENT TO PARTICIPATE}

Not applicable.

\section{CONSENT FOR PUBLICATION}

Not applicable.

\section{ADDITIONAL INFORMATION}

Supplementary information The online version contains supplementary material available at https://doi.org/10.1038/s41416-021-01688-0.

Correspondence and requests for materials should be addressed to Shun-Ichiro Kageyama.

Reprints and permission information is available at http://www.nature.com/ reprints

Publisher's note Springer Nature remains neutral with regard to jurisdictional claims in published maps and institutional affiliations.

\footnotetext{
Open Access This article is licensed under a Creative Commons Attribution 4.0 International License, which permits use, sharing, appropriate credit to the original author(s) and the source, provide a link to the Creative Commons license, and indicate if changes were made. The images or other third party material in this article are included in the article's Creative Commons license, unless indicated otherwise in a credit line to the material. If material is not included in the article's Creative Commons license and your intended use is not permitted by statutory regulation or exceeds the permitted use, you will need to obtain permission directly from the copyright holder. To view a copy of this license, visit http://creativecommons. org/licenses/by/4.0/.
}

(c) The Author(s) 2022 